\title{
Synthesis of N,N'-bis (a-methylsalicylidene)-3,4'-diaminodiphenyl ether
}

\section{A. A. Jarrahpour*, S .Rezaei}

Department of Chemistry, College of Sciences, Shiraz University, Shiraz 71454, Iran tel: +98 711 2284822, fax: +98 711 2280926, e-mail: jarrah@chem.susc.ac.ir and aliasghar6683@yahoo.com

*Author to whom correspondence should be addressed

Received: 21 September 2005 / Accepted: 15 January 2006 / Published: 22 January 2006

Keywords: 2-Hydroxyacetophenone, 3,4'- diaminodiphenyl ether, Schiff base.

There have been few reports about the synthesis and application of Schiff-base ligands derived from 2-hydroxyacetophenone [1]. In order to investigate the electronic, steric and geometric effect of a methyl group on an imine carbon on asymmetric catalytic reactions, 2-hydroxyacetophenone (1) has been chosen as starting material for synthesizing Schiff-base ligands [2]. Suhai and his coworkers have studied the conformational effects on the proton affinity of the Schiff base in bacteriorhodopsin [3]. Jacobsen and his coworkers have studied about asymmetric catalysis of hetero-ene reaction with tridentate Schiff base Cr(III) complexes[4].<smiles>CC(=O)c1ccccc1O</smiles>

2<smiles>C/C(=N\c1ccc(Oc2cccc(/N=C(\C)c3ccccc3O)c2)cc1)c1ccccc1O</smiles>

3
1

2-Hydroxyacetophenone 1 (0.272 g, $0.24 \mathrm{~mL}, 2 \mathrm{mmol})$ and 3,4'-diaminodiphenylether 2 (0.33 g, $1 \mathrm{mmol})$ were dissolved in $10 \mathrm{~mL}$ of warm ethanol. The reaction mixture was refluxed for $10 \mathrm{~h}$ and allowed to stand aside. The solid crystals were filtered off and washed with ethanol.The pure Schiff base $\mathbf{3}$ was isolated as a light yellow crystalline solid (yield 78\%).

Melting Point: $182-184^{\circ} \mathrm{C}$

IR $\left(\mathrm{KBr}, v, \mathrm{~cm}^{-1}\right): 3244(\mathrm{OH}), 1620(\mathrm{C}=\mathrm{N})$.

${ }^{1} \mathrm{H}-\mathrm{NMR}\left(250 \mathrm{MHz}, \mathrm{CDCl}_{3}\right): \delta=1.65\left(6 \mathrm{H}, \mathrm{s}, \mathrm{CH}_{3}\right), 6.21(2 \mathrm{H}, \mathrm{d}, \mathrm{Ar}), 6.24(2 \mathrm{H}, \mathrm{d}, \mathrm{Ar}), 6.88-7.64(5 \mathrm{H}, \mathrm{m}$, Ar), 7.94(2H, d, Ar), 14.57(2H, s, OH).

${ }^{13} \mathrm{C}-\mathrm{NMR} \quad\left(62.9 \mathrm{MHz}, \quad \mathrm{CDCl}_{3}\right): \delta=17.22 ; 111.71 ; 113.20 ; 118.22 ; 119.63 ; 126.66 ; 128.88 ;$ $132.95 ; 142.73 ; 145.60 ; 146.60 ; 162.15 ; 171.23$.

MS (m/z): 436 (87\%), 317 (40\%), 210 (48\%), 139 (38\%), 91 (90\%). 
The authors thank the Shiraz University Research Council for financial support (Grant No.84-GR-SC-23)

\section{References:}

1. (a) Zhang, J. X.; Zhou, Y.; Cai, G. J. Mol. Catal, 1997, 11, 41-44; (b) Holland, D; Laidler, D.A.; Milner, D.J. J. Mol. Catal, 1981, 11,119-127. (c) Chen, G. M.; Chen, F.; Zhou, C, Chem. Chin. Univ, 1995, 16, 216-219. (d) Qiu, M.; Liu, G.; Yiao, X, Chin. J.Catal, 2001, 22, 77-80. (e) Li, C.; Zhang, W.; Yao, X, Chin. J, Catal. 2000, 21, 77-80. (f) Li, Z. N.; Liu, G.; Zheng, Z. Tetrahedron 2000, 56, 7187-7191.

2. Gao, W. T.; Zheng, Z.; Molecules 2002, 7, 511-516.

3. Tajkhorshid, E.; Paizs, B.; Suhai, S. J. Phys. Chem. 1997, 101, 8021-8027.

4. Ruck. R. T.; Jacobsen E. J. J. Am. Chem. Soc. 2002, 124, 2882-2883.

Sample Availability: Available from MDPI.

(C) 2006 MDPI. All rights reserved. 